\title{
Modelo informático integrado AmI-IoT-DA para el cuidado de personas mayores que viven solas
}

\section{Integrated computer model of AmI-IoT-DA for care of elderly people living alone}

\author{
Andrés Armando Sánchez ${ }^{1}$ (D) Enrique González Guerrero $^{1}$ (D) Luis Eduardo Barreto $^{1}$ (iD \\ ${ }^{1}$ Facultad de Ingeniería, Pontificia Universidad Javeriana, Bogotá, Colombia \\ asanchez-m@javeriana.edu.co, egonzal@javeriana.edu.co, luis.barreto@javeriana.edu.co
}

(Received: 12 February 2019; accepted: 22 February 2019)

\begin{abstract}
Resumen
Las personas de la tercera edad sufren deterioro físico y mental que les impiden y/o dificultan el control de las tareas del hogar, la pérdida de su independencia y autonomía, lo que afecta su calidad de vida y bienestar. Este artículo presenta un modelo integrado AmI-IoT-DA en capas que integra funcionalidades de Internet de las Cosas (IoT), Inteligencia Ambiental (AmI) y data analytics (DA). El modelo se aplica al monitoreo y asistencia de las personas de la tercera edad que viven solas. Además, plantea cuatro segmentos encargados de automatizar la vivienda, supervisar al usuario, tomar decisiones, supervisar eventos, identificar hábitos, y acceder a servicios AmI, IoT y Data Analytics.

Palabras clave: Internet de las cosas, Inteligencia Ambiental, Analítica de Datos, cuidado adulto mayor, bienestar.

Abstract

Elderly people suffer physical and mental deterioration, which they prevent and / or hinder the control of household chores, the loss of their dependence and autonomy, affecting their quality of life and wellbeing. This paper presents an AmI-IoT-DA integrated layered model integrating functionalities of the Internet of Things (IoT), Ambient Intelligence (AmI) and Data Analytics (DA). The model is applied to the monitoring and assistance of elderly people living alone. The model proposes four segments responsible for automating housing, supervising the user, taking decisions, supervise events, identify habits, and access AmI, IoT and Data Analytics services

Keywords: Internet of Things, Environmental Intelligence, Data Analytics, adult care, welfare.
\end{abstract}

\section{Introducción}

Una de las problemáticas que enfrentan las personas de la tercera edad (adultos mayores) que viven solas, es que presentan dificultades en el desarrollo de actividades cotidianas debido a su condición natural y al proceso de envejecimiento de su cuerpo (Organización de la Naciones Unidas, 2006), las cuales se manifiestan en síntomas de deterioro que afectan su calidad de vida y su razonamiento. Las principales dificultades que presentan son: la disminución de la capacidad física; la capacidad cognitiva; la capacidad de controlar las funciones fisiológicas; pérdida de sus sentidos; entre otras (Felipe Salech, Rafael Jara, \& Luis Michea, 2012). Debido a lo anterior, requieren de un cuidador en el hogar para el desarrollo de sus actividades según su nivel de autonomía; esta persona debe cuidar, apoyar, acompañar y ayudar en lo que necesiten; sin embargo, esto no se cumple totalmente en el contexto colombiano. Sumado a esto, para el año 2020 se calcula que, por cada 100 adolescentes menores de 15 años, habrá 50 personas mayores de 60 años. Además, proyecta una expectativa de vida de 75 a 80 años, por lo que se entiende que existirán más personas de la tercera edad solos y/o abandonados en su hogar (Organización Mundial de la Salud, 2015).

Las personas de la tercera edad, además de estar solos y enfermos, deben asumir costos de 
desplazamiento, enfrentar problemas de tráfico, inseguridad y el riesgo de contagio de enfermedades, al tener que asistir a centros hospitalarios congestionados, para chequeos de rutina y tratamientos de sus enfermedades (Morelos Ramírez, Ramírez Pérez, Sánchez Dorantes, Chavarín Rivera, \& Meléndez-Herrada, 2014). Dependiendo de la gravedad de la enfermedad, en muchos casos el tratamiento puede ser realizado de manera ambulatoria o remota en casa (Lopardo et al., 2015); lo que trae como beneficio la salud de los pacientes, descongestión de los centros hospitalarios y, por último, permite aprovechar mejor el tiempo de los médicos y los especialistas tratantes (Gómez García \& Velasco Medina, 2014). Para dar solución a la problemática del adulto mayor que vive solo, se han realizado aproximaciones diseñadas para el cuidado y monitoreo en casa como redes de sensores inalámbricos (Villar-Montini, 2009); monitoreo remoto en el hogar vía celular (Chandler, 2014); realidad aumentada usando dispositivos móviles (González, Villegas, Ramírez, Sánchez, \& Domínguez, 2014); recolección de análisis de señales biomédicas (Campaña Bastidas \& Londoño Peláez, 2013); y dispositivos médicos remotos (Rajkomar, Mayer, \& Blandford, 2015). Algunas de estas tecnologías soportan parametrización remota (Fanucci et al., 2013), comunicación satelital (Guevara Valdivia et al., 2011); integración con sistemas de información geográficos (Parra-Henao, 2010).

Otras soluciones han estado encaminadas en brindar bienestar al adulto mayor, entre estas el planteado por Hassanalieragh, que define las oportunidades y retos existentes en una solución de monitoreo de las condiciones de salud implementando Internet de las Cosas (IoT) como tecnología de impacto (Hassanalieragh et al., 2015); Zamora -Izquierdo, Santa, \& Gomez-Skarmeta (2010) proponen DOMOSEC, que es una solución dada desde la domótica para la automatización del hogar y que podría usarse para el monitoreo de personas, usando dispositivos comerciales; Mileo, Merico, \& Bisiani (2008) plantean el uso de redes de sensores inalámbricos (WSN) en las cuales soportan un sistema de "casa inteligente" para el cuidado de ancianos; Doğali Çetin, Çetin, \& Bayimiş (2015) plantean una arquitectura para el tele-cuidado que monitorea signos vitales como el Electroencefalograma (EEG) y Electrocardiograma (ECG) en tiempo real para la atención oportuna de complicaciones médicas en pacientes de la tercera edad; Silva, Mouttham, \& El Saddik (2009) plantean Unimeds, un sistema que se apoya en la inteligencia ambiental para el suministro y control de medicamentos a personas que son asistidos en su hogar. Estos trabajos demuestran el gran potencial que existe para brindar bienestar y monitorear a adultos mayores con las tecnologías existentes.

Para garantizar de manera remota el cuidado del adulto mayor, se han desarrollado metodologías que permiten el levantamiento de requerimientos (González Palacio \& Urrego Giraldo, 2010), sistemas de transmisión, consolidación y procesamiento de señales biomédicas (Doğali Çetin et al., 2015), sistemas de apoyo a la toma de decisiones médicas (Celler \& Sparks, 2015); y sistemas de generación de alertas (Skubic, Guevara, \& Rantz, 2015). Estos trabajos vistos en conjunto definen los requerimientos mínimos que se deben cumplir en el diseño de cualquier sistema de tele-cuidado orientado a los adultos mayores.

En este artículo se presenta la integración de los modelos de referencia de Internet de las Cosas (IoT), Inteligencia Ambiental (AmI) y Analítica de Datos (DA), en un modelo informático integrado llamado Quysqua, y la relevancia que tiene en aplicaciones de tele-cuidado de adultos mayores. Esta integración está dividida en dos dimensiones: la primera, se enfoca en el cuidado y bienestar de personas de la tercera edad sanos o enfermos con cuidados especiales en casa; con lo que se pretende tener un marco común que brinde la posibilidad de mejorar la calidad de vida, bienestar y cuidado médico remoto en su hogar. La segunda dimensión se enfoca en el ámbito informático y presenta la viabilidad técnica de integración en un único modelo informático que provea un acompañamiento al adulto mayor, a los familiares y al personal médico, incorporando y profundizando el componente de herramientas para analítica (DA) en un ambiente de aplicación de IoT, AmI y DA. En este artículo se presenta: la definición del caso de referencia que especifica la problemática y su entorno, el diseño del modelo integrado Quysqua, la arquitectura del prototipo que implementa el modelo y las estrategias de validación del modelo y el prototipo.

\section{Definición del Caso de Referencia}

Para el diseño del modelo Quysqua se especifica un caso de referencia. Este mismo caso de referencia se utiliza para parametrizar un sistema multi-agente (SMA) que se utiliza para validar el modelo. Se define como caso de referencia a la implementación del modelo en la asistencia de cuidado y generación de bienestar a una persona de la tercera edad que vive sola en una unidad residencial (ambiente), y a los roles definidos y su interacción. El caso de referencia fue definido inicialmente por Agreda Chamorro, (2015).

\subsection{Definición del ambiente}


El ambiente en el que se aplica el modelo es un hogar con elementos básicos de una vivienda, muebles y electrodomésticos, componentes tecnológicos de domótica e instrumentos de tele-cuidado. Dentro de los componentes básicos de vivienda se encuentran componentes físicos de delimitación como: pisos, escaleras, muros, techos, ventanas y puertas, que definen el límite, la división y el acceso de la vivienda; áreas de uso e infraestructura sanitaria y de servicio como: servicios públicos, sistema de iluminación, control de ambiente y servicios médicos en casa. Estos elementos deben ser controlados y monitoreados para garantizar el confort y bienestar de adulto mayor. Por último, cabe mencionar que en este primer modelo de validación se asume que se conoce su distribución y ubicación de los elementos de la unidad de residencia, muebles, electrodomésticos, y demás implementos que el individuo tenga en su hogar, y que adicionalmente se tiene la información de los electrodomésticos con capacidad de conexión, y se conoce su protocolo y estándar de conexión.

En el ambiente definido se pueden generar eventos que se deben monitorear para garantizar el bienestar del adulto mayor. Los eventos de bienestar se clasifican en tres clases: médico, físico y emocional. Como se muestra en la Figura 1, los eventos de bienestar son generados a partir de parámetros clasificados según el tipo de interacción que el adulto mayor tiene con el ambiente.

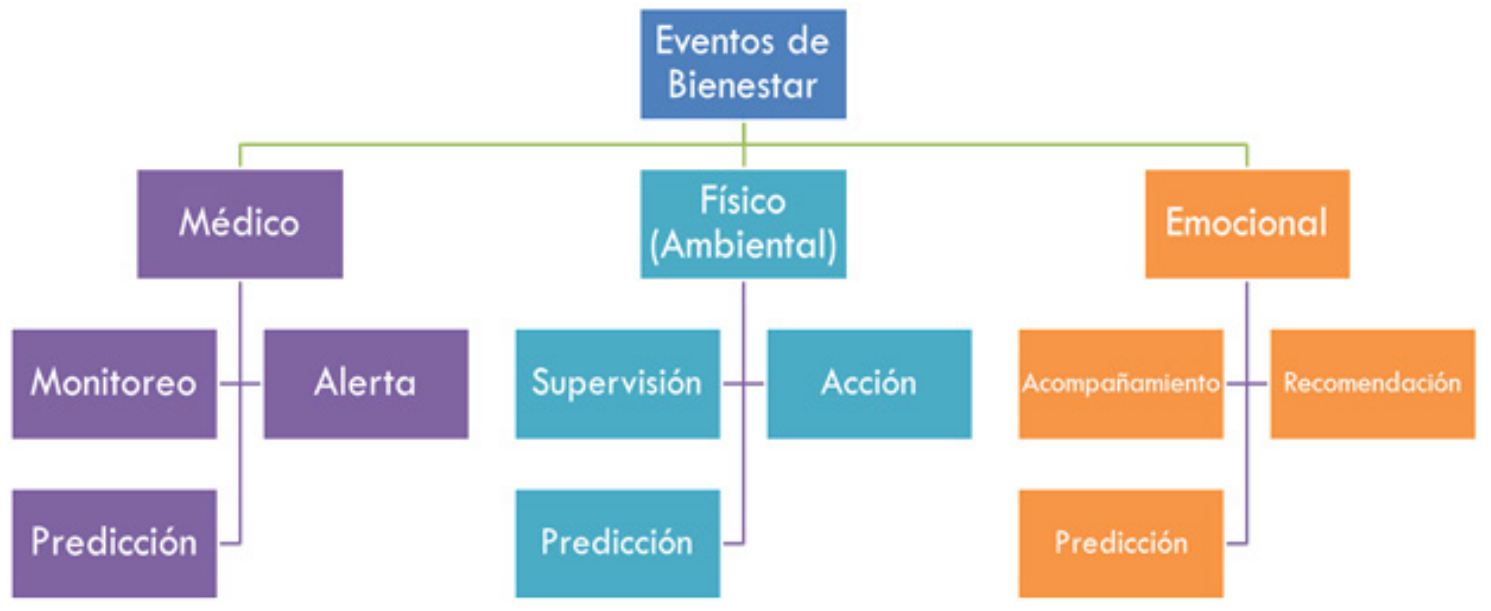

Figura 1. Clasificación de los eventos de bienestar

Médico: los eventos médicos son aquellos que se generan por la supervisión del usuario y se clasifican en cuatro: monitoreo de los signos vitales, cambios en su estado físico, frecuencia de ejercicio físico y cognitivo, ingesta de medicamentos y salidas del ambiente por razones médicas. Los eventos de alerta son el abastecimiento de medicamentos, el horario de ingesta de medicamentos, la sugerencia de terapias físicas o cognitivas, el recordatorio de agenda médica y las urgencias que se generan por un botón de alerta médica o automática, según el estado de los signos vitales. Por último, los eventos de predicción son mensajes de información con posibles cambios del estado de salud del usuario y nivel de autonomía, realizándose con el análisis de tendencias de eventos de monitoreo y alerta.

Físico (ambiental): los eventos ambientales son aquellos que se generan por la supervisión de la vivienda y la interacción con el usuario, se clasifican en cinco tipos: 1) eventos de supervisión, que incluyen las salidas eventuales del usuario de la vivienda y los hábitos de comportamiento en la vivienda; 2) eventos alimenticios, que incluyen los cambios en sus hábitos fisiológicos y nutritivos y que a su vez comprenden el uso de los elementos de la vivienda, la seguridad del ambiente respecto a gases, inundaciones o incendios, 3) eventos de confort, que incluye medidas de la temperatura, la humedad, el ruido y la luz; 4) eventos de acción, son los eventos que actúan sobre el ambiente: como la automatización domótica del mismo, alertas de seguridad, y recomendaciones al usuario para interactuar con la vivienda o de sus hábitos; y 5) eventos predictivos, que pueden ser el cambio de hábitos del usuario o los cambios de estado del ambiente.

Emocional: los eventos emocionales son aquellos que se generan por la interacción del usuario con usuarios que tienen otros roles como familiares y personas cercanas; se clasifican en cuatro tipos: 1) acompañamiento, que incluye las salidas recreativas del usuario al exterior, la convivencia, encuentros, y comunicación con otros; 2) los eventos de recomendación son los relacionados a motivar al usuario a comunicarse con otros o hacer actividades de entretenimiento como ver televisión, escuchar música, entre 
otros. Estas recomendaciones se pueden priorizar por gusto o frecuencia; y 3) los eventos de predicción que se pueden dar son el posible cambio del estado emocional y sus hábitos de comunicación e interacción.

\subsection{Roles}

Para el caso de referencia se definieron roles. Estos roles definen sus propios requerimientos, lo que hace que al momento de diseñar el modelo se tengan en cuenta. Estos roles se pueden ver en la Figura 2.

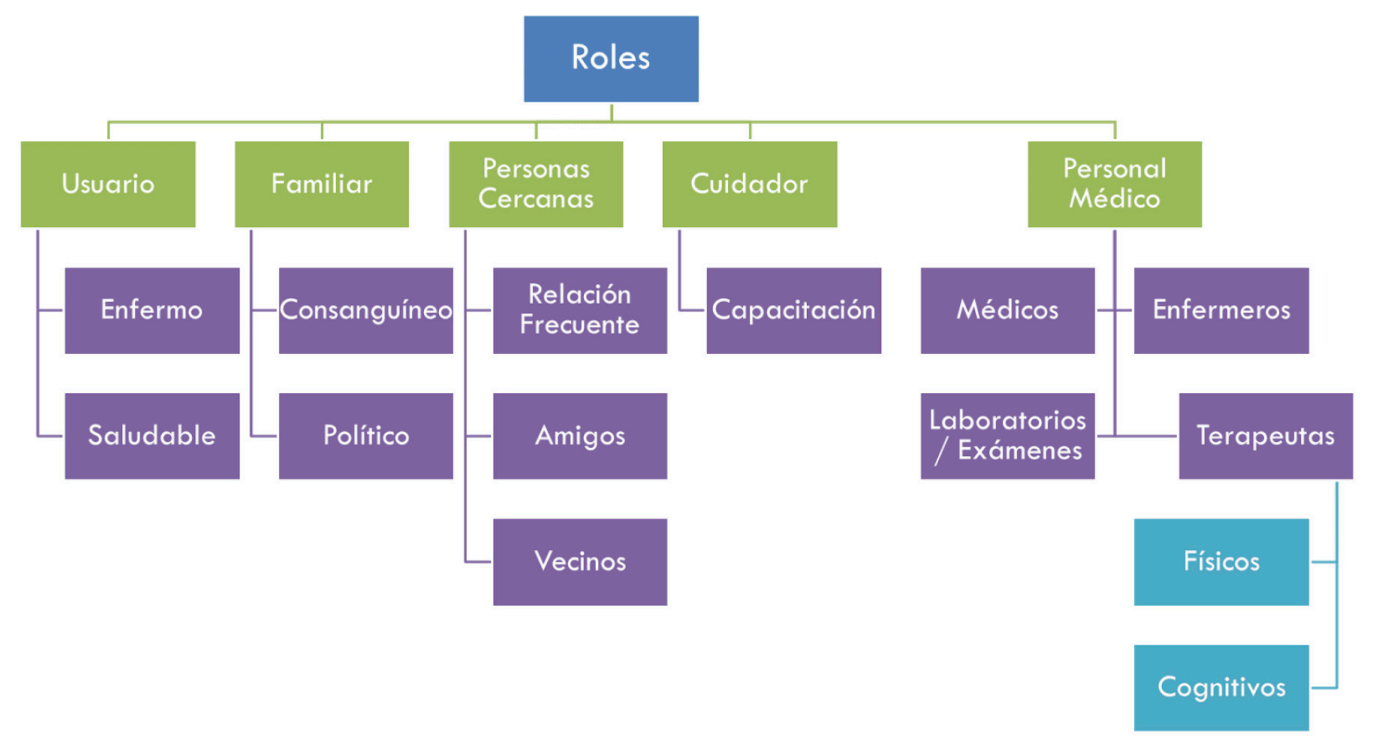

Figura 2. Definición de roles de usuarios del caso de referencia

Usuario: es una persona mayor de 60 años en etapa de vejez, que es una condición humana y no una enfermedad; por lo anterior, los usuarios se dividen en saludables y enfermos. Las enfermedades que aquejan a los usuarios impactan el grado de autonomía que ellas tienen para interactuar con su entorno. Si se tiene en cuenta que las personas de este rol viven solas, se les debe brindar asistencia para apoyarlas en sus actividades cotidianas. A los usuarios se les puede monitorear los signos vitales, niveles de bienestar, las actividades realizadas y recomendar actividades.

Familiar: los familiares son un factor importante para el estado de ánimo del usuario, sin importar si es familia consanguínea o política. La interacción constante del usuario con ellos lo motiva y anima y, en consecuencia, evita que entre en un estado de depresión, siendo esta una de las causas de deterioro médico más comunes. De los familiares se puede monitorear las interacciones con el usuario por cualquier medio e informar el estado del usuario.

Personas cercanas: de igual forma que los familiares, las personas cercanas al usuario lo motivan y animan. Estas personas son con quienes tiene una interacción frecuente al realizar sus actividades cotidianas como caminar, hacer ejercicio, comprar víveres, socializar, entre otras. Pueden ser vecinos, amigos y/o personal de tiendas. De ellos se puede monitorear las interacciones con el usuario, y si son el motivo de una salida del usuario de la vivienda.

Cuidador: es la persona capacitada que se encarga de asistir al usuario cuando este no pueda cumplir autónomamente alguna actividad. Si el usuario tiene un grado de autonomía alto, este puede ser su propio cuidador, si no, este podría ser: un personal dedicado a este oficio, un familiar, una persona cercana o el personal médico. A ellos se les reporta el estado de salud y físico del usuario, recordatorios de las actividades que debe cumplir, estado del ambiente e información que apoye su labor de asistencia al usuario.

Personal médico: está compuesto por enfermeros, especialistas, terapeutas, médicos y laboratorios. El responsable del diagnóstico, medicación y seguimiento del estado físico y médico del usuario es el médico tratante. Al médico tratante se le debe reportar la información del estado médico, datos vitales y estado físico; este autoriza al resto del personal a entrar en acción. 


\section{Descripción general del modelo}

El modelo se plantea en cuatro grandes segmentos en pila: segmento IoT (segmento de internet de las cosas), segmento AmI (segmento de inteligencia ambiental), segmento DA (segmento Data Analytics) y segmento de ApS (segmento de aplicación). Cada segmento agrupa funcionalidades encapsuladas en capas, componentes y subcomponentes, como se muestra en la Figura 3. Si bien en el modelo se presentan los segmentos y capas en pila, en su implementación los segmentos, capas, componentes y subcomponentes son independientes y modulares, así, dependiendo de la aplicación donde se implemente el modelo no será obligatorio implementar todas las capas y componentes.

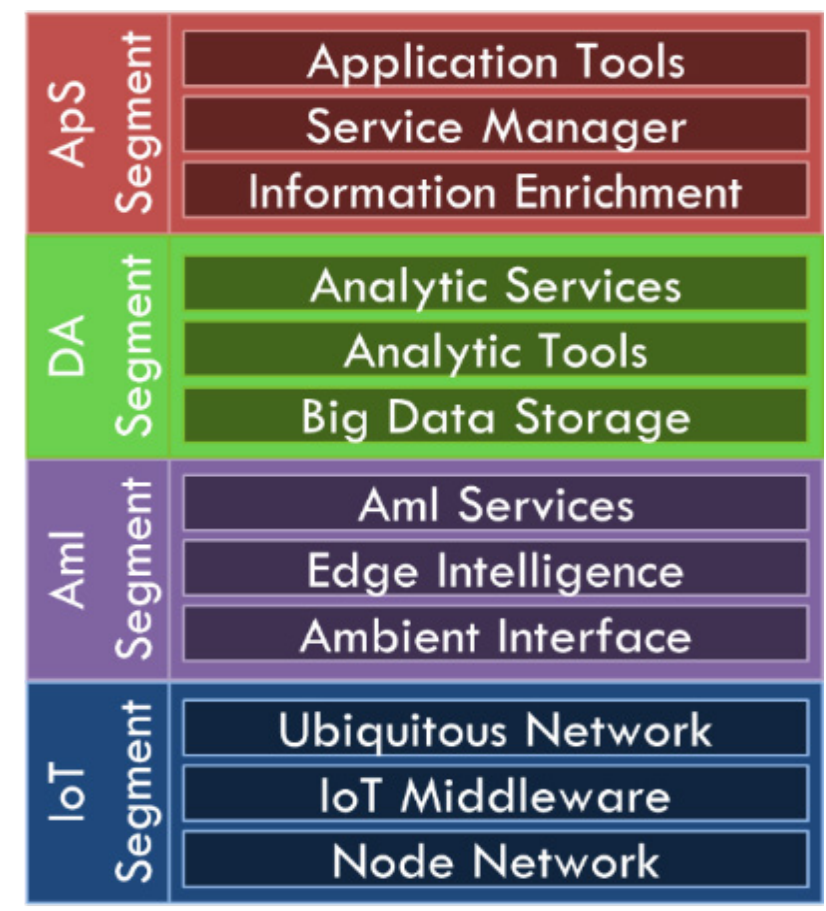

Figura 3. Modelo informático integrado AmI-IoT-DA Quysqua

En el segmento IoT se agrupan las capas encargadas de las funcionalidades de configuración de redes de nodos (sensores y actuadores), integración de tecnologías de comunicación heterogéneas, y gestión de datos de sensores (homogeneizar, transformar y validar) y envío de órdenes a actuadores como servicios ubicuos. En este segmento se hace la automatización de la vivienda, creando redes de nodos específicas para supervisar y controlar la vivienda, y la monitorización del usuario; de esta manera, se brinda una plataforma que se encarga de la integración y comunicación con los dispositivos físicos de la vivienda y el usuario y la transmisión a un integrador de borde.

En el segmento AmI es donde están las capas encargadas del consumo de datos suministrados por el segmento IoT, la aplicación de una inteligencia de borde para la toma de decisiones, y generación de servicio de envío y recepción de mensajes por una interface $A m I$ de comunicación. En este segmento se hace la interpretación del estado de la vivienda, y el usuario a partir de los datos enviados por los sensores ambientales y wearables cambia el estado de la vivienda a partir de los actuadores publicados como servicios ubicuos, interactuar con el usuario, y prestar servicios de generación de mensajes tipo notificación y/o alerta a otros roles.

El objetivo del segmento $D A$ es realizar un análisis a largo plazo y más exhaustivo de los datos recuperados de los segmentos inferiores $(I o T$ y $A m I)$. Los resultados generados por esta capa pueden ser accesibles a los usuarios como información de soporte para tomar decisiones de alto nivel. El segmento $D A$ se compone de tres capas. La capa inferior está dedicada al almacenamiento, limpieza y organización de los datos. La segunda capa incorpora un conjunto de herramientas diferentes para realizar el análisis de datos, la mayoría de ellas basadas en técnicas de aprendizaje automático. La capa superior incluye una interfaz de servicio que se utiliza para acceder a los servicios proporcionados por los segmentos desde la capa de aplicación superior. 
Finalmente, El Segmento ApS es el encargado de proveer un conjunto de servicios adaptados de $I o T$ y/o $A m I$ y/o $D A$, visualización y presentación de la información y un conjunto de herramientas para desarrollar aplicaciones. En este segmento, se ofrecen los datos de la vivienda y del usuario como servicios adaptados según el rol y el dispositivo de acceso, el control de acceso, y las herramientas necesarias para la construcción de aplicaciones cliente de consumo y/o análisis para cada rol.

\section{Implementación del modelo}

Se desarrolló un prototipo que implementa el modelo propuesto y se ajusta al caso de referencia descrito. En esta sección se explica con detalle cómo se implementó el modelo propuesto, empezando por el análisis y elaboración de un modelo de sistema multi agente (SMA), luego el diseño y la elección de las tecnologías que se tuvieron en cuenta, finalizando con el uso de un estilo de arquitectura de software basado en capas.

\subsection{Modelo de sistema multi agente (SMA)}

Para realizar el SMA que ayudará en la validación del modelo Quysqua a través de la validación del prototipo implementado, se diseñó un modelo de sistemas multi agentes (SMA). Para realizar el diseño del sistema multi agente, se definen actores a partir de los roles definidos en el caso de referencia, los cuales se convierten en base del análisis del sistema de inteligencia ambiental y toma de decisiones, al igual que en lectura y acción en el ambiente. Luego, se debe hacer un análisis de metas del sistema, basado en las temáticas previamente descritas en el caso de referencia, y el análisis documental de las propuestas de solución. Para finalizar, se definieron los roles y las interacciones necesarias para proponer la arquitectura del SMA enmarcada. Una vez propuesto el SMA, se define cual es la inteligencia que debe tener cada uno de los agentes a partir de los objetivos de cada uno.

En la Figura 4 se expone el diagrama de interacciones del sistema multi agente, el cual se lee de izquierda a derecha. Se organizó según los segmentos y capas del modelo Quysqua; en donde los óvalos representan los agentes, las flechas blancas las interacciones, el texto sobre las flechas blancas los protocolos (request - response y/o publish suscribe) y los triángulos amarillos los adaptadores. A continuación, en la Tabla 1 se describe la función de cada agente.

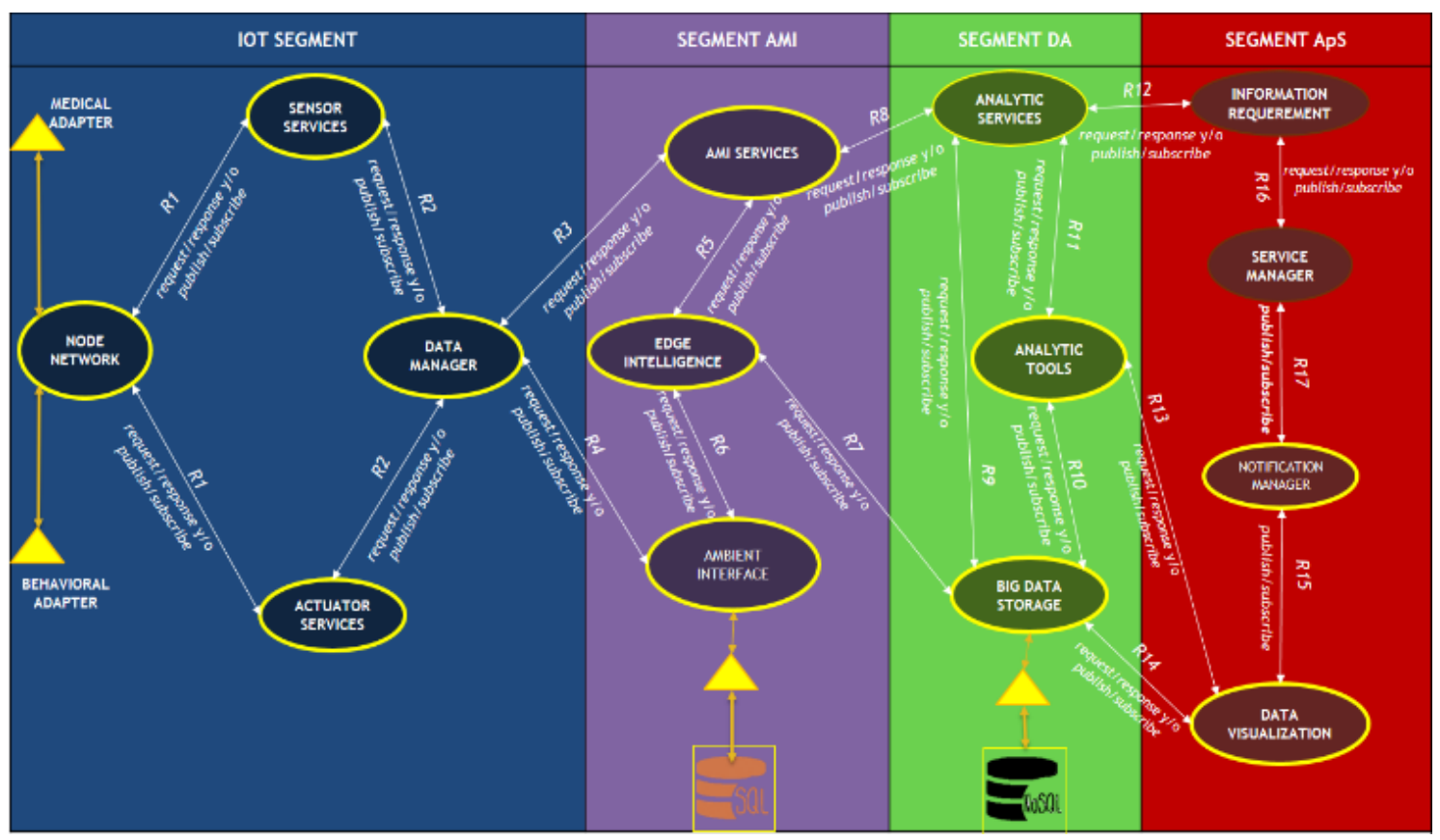

Figura 4. Modelo de sistema multi agente (SMA) 
Tabla 1. Descripción SMA.

\begin{tabular}{|c|c|}
\hline Agente & Descripción \\
\hline Node Network & Interactúa con el ambiente tanto en la recepción como en actuación a través de los adaptadores. \\
\hline Sensor Service & $\begin{array}{l}\text { Manejador de sensores para gestionar la lectura de datos, en sensores síncronos para los cuales } \\
\text { se solicita lectura. }\end{array}$ \\
\hline Actuator Service & Convierte en eventos las señales de cada uno de los sensores o actuadores que tengan a cargo. \\
\hline Data Manager & $\begin{array}{l}\text { Recibe los mensajes de los sensores y actuadores, y realiza un proceso de homogeneización y } \\
\text { transformación de datos para generar un mensaje más limpio. }\end{array}$ \\
\hline Edge Intelligence & $\begin{array}{l}\text { Toma las decisiones sobre el ambiente y el usuario, la generación de conocimiento y servicios } \\
A m I \text {, la interacción con el usuario y se realiza un primer nivel de analítica de datos. }\end{array}$ \\
\hline AmI Service & $\begin{array}{l}\text { Envía las acciones hacia el segmento } I o T \text { y las notificaciones hacia agentes de segmento } D A \text { o } \\
\text { Aps. }\end{array}$ \\
\hline $\begin{array}{l}\text { Ambient } \\
\text { Interface }\end{array}$ & $\begin{array}{l}\text { Almacena las reglas reactivas y persiste en la base de datos todo lo que genera el segmento } \\
\text { AmI. }\end{array}$ \\
\hline Analytic Services & Genera la notificación del segmento $D A$ hacia el segmento $A m I$ y el segmento Aps. \\
\hline Analytic Tools & $\begin{array}{l}\text { Realiza el modelado de los hábitos de los usuarios, crea reglas de sugerencia e interpretación de } \\
\text { resultados que ejecuta el Supervisor de Eventos analítica. }\end{array}$ \\
\hline Big Data Storage & $\begin{array}{l}\text { Almacena las reglas deliberativas y persiste en la base de datos todo lo que genera el segmento } \\
D A \text {. }\end{array}$ \\
\hline $\begin{array}{l}\text { Notification } \\
\text { Manager }\end{array}$ & $\begin{array}{l}\text { Obtiene las notificaciones de los distintos agentes y genera un mensaje externo con el contenido } \\
\text { de este a través de representaciones gráficas (diagrama) o escritas (email). }\end{array}$ \\
\hline $\begin{array}{l}\text { Data } \\
\text { Visualization }\end{array}$ & $\begin{array}{l}\text { Crea una capa de presentación a través de una interfaz gráfica GUI para que un usuario acceda } \\
\text { a la información a través de gráficos y tablas. }\end{array}$ \\
\hline Service Manager & Se encarga de brindar la seguridad al modelo de agentes mediante un Access control service. \\
\hline $\begin{array}{l}\text { Information } \\
\text { Requirement }\end{array}$ & $\begin{array}{l}\text { Realiza todo lo relacionado con la adaptación de la información y contenidos de los mensajes } \\
\text { recibidos del segmento } A m I \text { o } D A \text {. }\end{array}$ \\
\hline
\end{tabular}

\subsection{Diseño y tecnologías del modelo propuesto}

El prototipo implementó parcialmente los componentes de las cuatro capas del modelo de la siguiente manera: para la implementación del segmento IoT, se tuvieron dos fuentes de datos; el primero es la base de datos PostgreSQL que almacenó el proyecto "Sistema de Consolidación y análisis de información biomédica SICOBIO” (Parra Alba, 2016) durante más de seis meses con información de señales biomédicas de pacientes adultos mayores del hospital San Ignacio que recibían cuidado médico en casa; el segundo son los datos generados por el simulador desarrollado por Agreda Chamorro (2015), el cual muestra a través de un SMA el ambiente de un adulto mayor que vive solo, y cómo este se ve enfrentado a diferentes cambios físicos y afectivos. Para ello se hizo el desarrollo de una aplicación web con tecnología Java EEJSF, que implementa la librería EclipseLink JPA2.1, cuyo fin era manejar la persistencia a una base de datos PostgreSQL. Se creó un WebService SOAP, para la publicación de los datos de los sensores que se conectaron mediante Mosquito (implementación de MQTT), y un middleware que recolectara datos de los agentes simulados, nodos físicos, y datos recolectados por dispositivos wearables.

Para la implementación del segmento $A m I$ se tuvieron que construir tres componentes importantes; uno para el manejo de la inteligencia de borde, otro que maneja las reglas reactivas y notificaciones $A m I$, y otro que haga la función de persistencia a una base de datos. Se modificó y complementó el prototipo desarrollado por Agreda Chamorro (2015). La implementación se hizo desarrollando una aplicación standalone con tecnología Java SE, que implementan las librerías: BESA3-ALPHA1, además de incluir la librería jfuzzy para el manejo de una lógica difusa en la inteligencia de borde. Se usó MySQL-JDBC para manejar la comunicación con una base de datos MySQL del agente ambient Interface. Para el agente encargado de las notificaciones fue creado un conector cliente de WebService SOAP, para consumir los servicios de la implementación del segmento IoT, y una interfaz para que se consuman las notificaciones por los segmentos $D A$ y ApS.

La implementación del segmento DA se creó con los componentes de Big Data Storage, Analytic Tools, y Analytic Services. Para facilitar la identificación de atipicidades entre pares de señales, se ha construido una funcionalidad que utiliza el algoritmo de segmentación DBScan, y que presenta los resultados en un plano cartesiano. Cada uno de los segmentos que genera el algoritmo es marcado y etiquetado con un color distinto. Para mayor facilidad en el análisis, el detalle de las alertas se presenta en la parte inferior de la pantalla. Esta funcionalidad le permite al especialista filtrar por cada uno de sus 
pacientes, y efectuar las combinaciones de señales que considere necesarias para sus análisis. Se hizo el desarrollo de una aplicación web con tecnología JavaEE-JSF, que implementa la librería de EclipseLink JPA2.1, para manejar la persistencia a una base de datos MySQL de analítica donde se consultan las alertas y notificaciones del segmento $D A$ y se consumen los datos del segmento $A m I$ a través de un cliente de WebService SOAP para la publicación de los servicios AmI e IoT.

Para la implementación del segmento ApS se crearon los componentes Notification Manager y Data Visualization. Para la visualización de los datos, se utilizaron las librerías de software libre chart de Primefaces y JFreeChart. Estas librerías, ofrecen capacidades de visualización de datos que pueden facilitar el seguimiento de pacientes y toma de decisiones mediante gráficos de líneas que representan el comportamiento o tendencia de una señal determinada, gráficos combinados para representar tendencias entre valores mínimos y máximos, y nubes de puntos para identificar la tendencia que muestra DBScan.

\subsection{Arquitectura de software de la aplicación}

En la implementación general del modelo propuesto, se construyó la arquitectura que se puede ver en la Figura 5. Esta arquitectura define el comportamiento e interacción entre las capas del modelo propuesto.

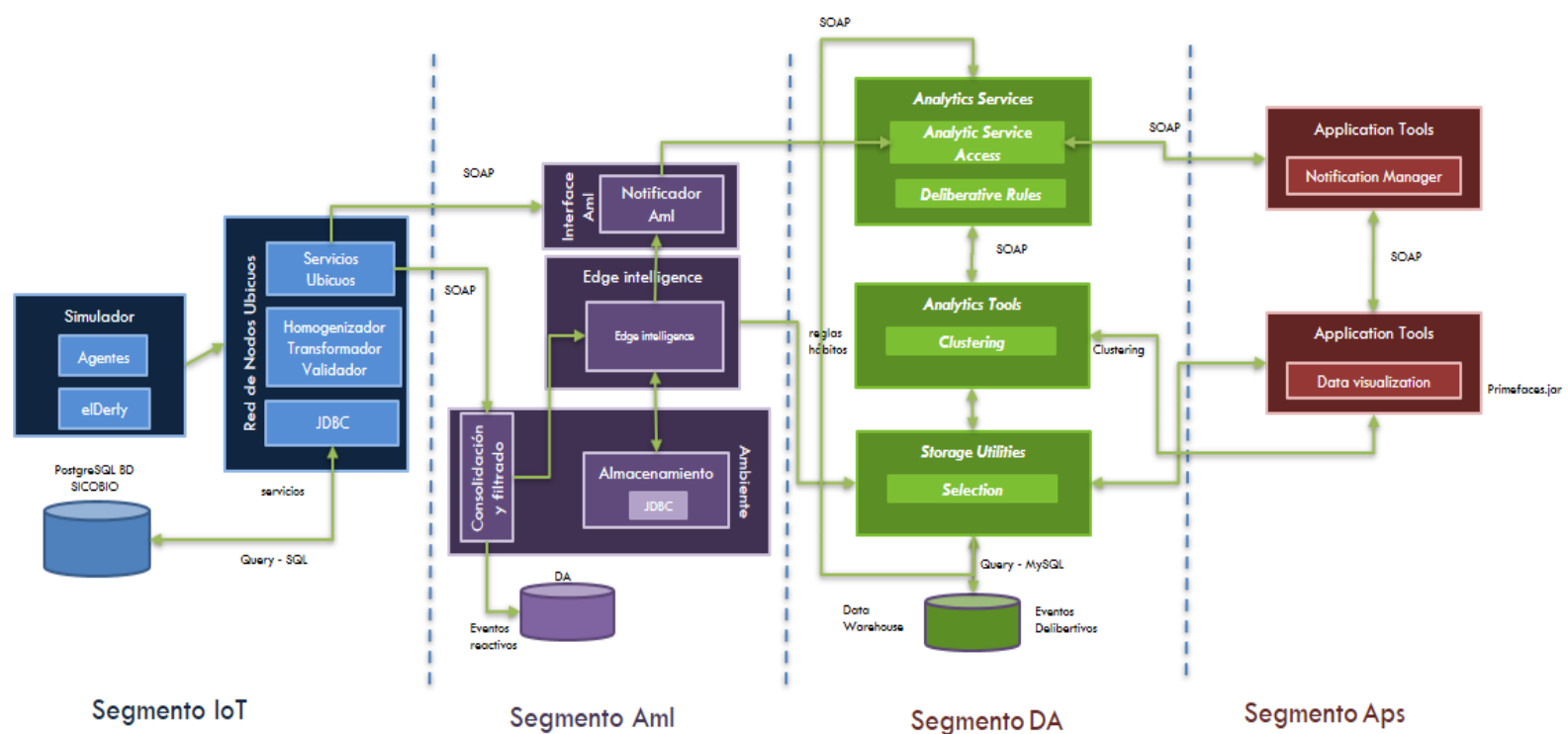

Figura 5. Arquitectura de software de la implementación

La arquitectura de software propuesta de este sistema computacional está definida por el estilo arquitectónico llamado "sistema en capas" (Sommerville, 1995), comprendida por los elementos de software, las propiedades visibles de estos elementos y las relaciones entre ellos. En la Figura 5, se muestra un diseño preliminar o de alto nivel de los cuatro segmentos con los cuales se construye el sistema implementado, en el cual se tuvieron en cuenta aspectos como: componentes (servidores, clientes, bases de datos, filtros, capas en un sistema jerárquico, etc.), protocolos de comunicación, interacciones entre segmentos (llamadas a procedimientos, protocolos $\mathrm{C} / \mathrm{S}$, protocolos de acceso a BD, etc), el incremento en el tamaño y complejidad del software, flujo de los datos, reutilización de estructuras asociadas a problemas similares, e implementación concreta de partes de otras estructuras.

Al estar enmarcado en el estilo arquitectónico de "sistema en capas", se asocia una serie de propiedades que lo caracterizan y que determinan sus ventajas e inconvenientes, condicionando la elección de uno u otro estilo. Para entender la arquitectura propuesta en el presente documento, en la Figura 5 se debe tener en cuenta las invariantes empleadas:

- Se dividió la arquitectura en cuatro segmentos que se leen de izquierda (Segmento IoT) a derecha (Segmento Aps); esto permitió entender el sistema global en términos de la combinación de capas, componentes y conectores, soportando de buena manera la reutilización y facilitando el mantenimiento, mejora y diagnóstico (rendimiento, deadlocks). 
- Organizado jerárquicamente en capas, donde cada capa provee servicios a la capa derecha y a su vez esta es servida por la capa izquierda.

- Los componentes son cada una de las capas, y cada componente lee las entradas, procesa la información y las transforma en salidas.

- Los conectores son los protocolos de interacción entre las capas.

- Una gran ventaja de la arquitectura propuesta es que se pueden cambiar las implementaciones, respetando las interfaces con las capas adyacentes.

A continuación, se detallan los componentes y conectores de los cuatro segmentos y/o capas:

Segmento IoT: los componentes simulador y red de nodos ubicuos se comunican al ambiente por medio de dos adaptadores. Un adaptador se comunica a una base de datos que tiene solo información médica del estado de salud del adulto mayor. La data se obtuvo a partir de la recolección de datos a través de dispositivos Fitbit (tecnología vestible como un reloj inteligente o un sensor en la camisa) que usaron los pacientes por un tiempo de aproximadamente un año. La otra base de datos entrega datos comportamentales del adulto mayor, los cuales son producto de simulaciones realizadas con agentes inteligentes. Así se enriquece el modelo con datos simulados y datos reales de aspectos médicos y comportamentales del adulto mayor. Tiene un esquema de comunicación Publish-Subscriber (este patrón yace en la capacidad que tienen los suscriptores de conocer el estado del sujeto, registrándose en un publicador para recibir notificaciones cuando algún evento en este último suceda) mediante servicios SOAP (Simple Object Access Protocol).

Segmento AmI: los componentes ambientes, edge intelligence, e interface AmI usan una base de datos donde se registran las reglas reactivas, los eventos del ambiente y usuario, y los datos que registra el agente de inteligencia de borde. Se comunican los datos mediante el protocolo SOAP que permite la comunicación entre aplicaciones a través un Servicio Web al que se puede acceder mediante protocolos Web estándar, utilizando XML. El registro de las alertas y notificaciones se realiza en la base de datos y las comunica a través de JPA-JDBC al segmento de DA.

Segmento DA: los componentes storage utilities, analytics tools, y analytics services usan un adaptador para consumir una base de datos Datawarehouse (a través de una ETL construida en SQL server management Studio). Esta se caracteriza por integrar y depurar información de los segmentos IoT y AmI para luego procesarla, permitiendo su análisis desde infinidad de perspectivas y con altas velocidades de respuesta. La creación de un datawarehouse representa el primer paso, desde el punto de vista técnico, para implantar una solución completa y fiable de Business Intelligence del proyecto planteado. En este almacén de datos se registran las reglas deliberativas, los resultados de los análisis de comportamientos y hábitos que genera el agente Analytic Tools; de igual forma, registra las notificaciones en la base de datos y las comunica a través de JPA-JDBC al segmento de Aps.

Segmento Aps: el componente application tools consume la misma base de datos del producto del segmento DA con JPA-JDBC para construir un dashboard (se usó el framework primefaces de JSF y la librería de java JFreeChart), el cual se usa como interfaz donde el usuario (médico, paciente o familiar) puede:

- Visualizar datos (gráficos, tablas, dahsboard) que pueden facilitar la interpretación y la toma de decisiones.

- Gestionar información para generar notificaciones a través de mensajes de texto, correo electrónico.

- Hacer un análisis sobre lo ocurrido, las recomendaciones dadas y el potencial impacto sobre el cuidado del adulto mayor.

\section{Validación y análisis de resultados}

Para validar la coherencia del modelo Quysqua, y para comprobar que se puede aplicar como solución de la problemática planteada y al caso de referencia descrito en la Sección 2, se plantean tres tipos de pruebas que se explican a continuación.

Prueba uno: respecto al caso de referencia. Se aplica el modelo Quysqua en una dimensión física cuando se asiste al cuidado del ritmo cardíaco del adulto mayor. Para el caso de referencia, en los eventos médicos, el ritmo cardíaco empieza en el segmento IoT, cuando se conectan los sensores wereables para medir el ritmo cardíaco del adulto mayor a través de señales como presión arterial sistólica y saturación de oxígeno. Luego, se dispone de una infraestructura que publica los datos que estos sensores arrojan. Además, se sirve de un componente para manejar las unidades de las señales en mmHG para la presión arterial sistólica y porcentaje (\%) para la saturación de oxígeno. A continuación, en el segmento AmI, se cuenta la inteligencia de borde que, según unas reglas reactivas, le informa al adulto mayor que debe tomar su 
medicamento todos los días a las 5pm, y si alguna señal no está en los márgenes normales se manda la acción al medio para que una alarma suene en el hogar y le recuerde tomar el medicamento. En el segmento $D A$, detecta los hábitos y diagnostica una anomalía; en consecuencia, da cuenta de que el paciente debe tomar el medicamento en otro horario, ante lo cual genera una notificación al segmento Aps donde el médico puede acceder a través de una aplicación web y tomar una decisión de cuál será la nueva frecuencia horaria con la cual el adulto mayor debe tomar el medicamento. Así se genera una nueva regla en el segmento AmI que obliga a tomar el medicamento a las $8 \mathrm{am}$. De este modo, se puede validar si la funcionalidad que propone cada capa se aplica coherentemente a la implementación puntual al caso de referencia.

Prueba dos: respecto a otros modelos ya reconocidos. Para probar que el modelo Quysqua, cumple con funcionalidades mínimas requeridas por los conceptos de data analytics, IoT e inteligencia ambiental, se eligieron tres trabajos en los cuales se desarrollaron modelos de referencia, y se compararon con el modelo propuesto. El objetivo de esta prueba es demostrar que el modelo abarca las funcionalidades de los modelos analizados. Los trabajos seleccionados fueron: Oracle (ORACLE, 2017), Cisco (CISCO, 2015), y NIST (Chang \& Grady, 2018). Como resultado de esta prueba se demostró que el Modelo Quysqua, define segmentos, capas, componentes, subcomponentes e interacciones, que fueron implementadas en trabajos relacionados, dándole validez y aplicabilidad al modelo propuesto. De igual manera, da relevancia, ya que el modelo propuesto puede ser usado como marco de referencia para implementaciones de $A m I$, IoT, domótica, WSN, data analytics, y telecuidado; esto trae como beneficio el ahorro de tiempo en la definición de componentes, funcionalidades básicas e interacciones.

Prueba tres: evaluación con el modelo de aceptación TAM. Para probar que el modelo Quysqua es coherente, completo, modular, relevante y aplicable, se presentó a cinco expertos en las áreas de data analytics, $I o T$ e inteligencia ambiental, tanto del ámbito empresarial como académico. Los expertos respondieron una encuesta de aceptación del modelo inspirado en TAM, que pretende medir si el modelo cumple con los componentes necesarios para soluciones en marcos de $D A$, $I o T$ y $A m I$, en el caso de referencia. Como resultado de esta tercera prueba, se evaluó el modelo en seis criterios. La evaluación del Modelo Quysqua, por parte de los cinco expertos, fue positiva. En promedio los expertos estuvieron de acuerdo en dar una buena o excelente calificación en los seis criterios en los que se evaluó el modelo propuesto.

Prueba cuatro: emulación del prototipo. Para probar el prototipo que implementa el modelo propuesto, se ejecutó una simulación de cuatro años de un ambiente que representaba el caso de referencia. Esta simulación se hizo con una herramienta basada en agentes propuesta por Agreda Chamorro (2015). De esta ejecución se pudo evidenciar que el prototipo era capaz de identificar patrones de comportamiento, hacer recomendaciones y generar analítica definida en el caso.

Con la validación y el análisis de los resultados se puede decir que el modelo es coherente, modular, aplicable, completo, relevante y compatible. En efecto, respecto al caso de referencia, se aplica perfectamente al ejemplo del adulto mayor, mostrando que es lo suficientemente completo para validar varias casuísticas o escenarios. De igual forma, se comprobó que, respecto a los modelos ya reconocidos, el modelo Quysqua implementa modularmente las tecnologías y principios de analítica de datos propuestos por grandes empresas como Cisco, Oracle o Nist. Los resultados de la evaluación de expertos que usaron la metodología TAM fue buena, pues todos los criterios quedaron por encima de 4 puntos sobre 5. En la simulación llevada a cabo se demuestra que sí cumple con la tarea de monitorización y analítica de los eventos, pero se espera desarrollar pruebas de aplicación práctica en el mundo real, en ambientes de cuidado de personas donde se interactúe con un adulto mayor. Para ver el detalle de las pruebas realizadas se puede consultar a Sánchez, González, \& Barreto (2018).

\section{Conclusiones}

El modelo resultante del proceso de integración fue un modelo amplio y robusto, que abarca los componentes y funcionalidades de $A m I$, IoT y $D A$. De igual manera, el modelo se planteó para que sea flexible y modular, de tal manera que las implementaciones que se hagan del mismo no necesariamente implementen todas las capas y componentes.

El modelo Quysqua propone una arquitectura por capas que se puede aplicar al caso de referencia propuesto, que se centra en el cuidado y bienestar de una persona de la tercera edad que vive sola en una unidad de vivienda. Las implementaciones de este modelo tendrían definida una arquitectura base, a la que solo se debe configurar las reglas de la inteligencia ambiental para garantizar el cuidado y bienestar. 
Para el proceso de evaluación del modelo propuesto se planteó una metodología que consiste en realizar cuatro pruebas. La comparación con productos desarrollados con anterioridad y el caso de referencia, más estas pruebas demostraron que el modelo es completo y aplicable en el tele-cuidado, además de ser compatible con otras definiciones $A m I$, IoT y $D A$. La evaluación del modelo por un grupo de expertos dio como resultado que el modelo es lo suficientemente maduro para ser implementado, además de contar con los atributos de calidad esperados. La última prueba, fue el desarrollo de un prototipo funcional y parcial del modelo propuesto. Este prototipo se usó para verificar que el modelo se puede implementar al caso de referencia y la aplicación de tele-cuidado. Si bien la prueba de operación con una perspectiva de largo plazo se hizo en un ambiente de simulación mediante un prototipo de implementación parcial del modelo, la parametrización del SMA se hizo con la definición de un caso de referencia realista, y el prototipo desarrollado respondió, generando alertas y notificaciones conforme al diseño.

Finalmente, es pertinente mencionar que cuando se validó el modelo en el sector empresarial, la respuesta en común fue satisfactoria. Ya que los evaluadores esperaban un prototipo funcional para probar sus técnicas y herramientas de analítica empresariales, configurando su ambiente de analítica de datos al caso de referencia propuesto. En efecto, algunas organizaciones se vieron interesadas en cómo se integra edge computing en una solución de data analytics. Esto representa una oportunidad de negocio.

Como trabajo futuro se sugiere la implementación de nuevos modelos de análisis que procesen la información obtenida de los diferentes sensores remotos de un paciente y dé a los profesionales de la salud nuevos elementos para la toma de decisiones.

También se sugiere enriquecer el prototipo con una App móvil, para que, mediante los sensores instalados en el Smartphone, obtenga y transmita datos de la actividad del paciente, y también, para que sirva de canal de comunicación entre el médico y el cuidador respecto a recomendaciones de tratamiento.

\section{Agradecimientos}

Agradecemos el apoyo de la Maestría en Ingeniería de Sistemas y Computación (MISyC) de la Facultad de Ingeniería, de la Pontificia Universidad Javeriana - sede Bogotá, por los recursos técnicos, logísticos y humanos que pusieron a nuestra disposición. A su vez, a los ingenieros Enrique Ruiz, Javier Agreda y William Parra, por su trabajo previo que sirvió como punto de partida para este trabajo.

\section{Declaración de conflicto de intereses}

Los autores declaran no tener conflicto de intereses con respecto a la investigación, autoría y/o publicación de este artículo.

\section{Referencias}

Agreda Chamorro, J. A. (2015). Diseño de un modelo de inteligencia ambiental para asistir a personas de la tercera edad. Pontificia Universidad Javeriana. Retrieved from http://pegasus.javeriana.edu.co/ PI133-01SisIntelAmbDiscap/

Campaña Bastidas, S. E., \& Londoño Peláez, J. M. (2013). Estudio de redes de sensores y aplicaciones orientadas a la recolección y análisis de señales biomédicas. Revista Gerencia Tecnológica Informática, 12(33), 85-99.

Celler, B. G., \& Sparks, R. S. (2015). Home Telemonitoring of Vital Signs-Technical Challenges and Future Directions. IEEE Journal of Biomedical and Health Informatics, 19(1), 82-91. https://doi.org/10.1109/ JBHI.2014.2351413

Chandler, D. L. (2014). Tomorrow's Hospital: How technology will be transforming both inpatient and at-home care. IEEE Pulse, 5(6), 16-21. https://doi.org/10.1109/MPUL.2014.2355298

Chang, W. L., \& Grady, N. (2018). NIST Big Data Interoperability Framework: Volume 1, Big Data Definitions [Version 2]. Retrieved December 12, 2018, from https://www.nist.gov/publications/nist-big-datainteroperability-framework

CISCO. (2015). Fog Computing and the Internet of Things: Extend the Cloud to Where the Things Are. Retrieved from https://www.cisco.com/c/dam/en_us/solutions/trends/iot/docs/computing-overview.pdf 
Doğali Çetin, G., Çetin, Ö., \& Bayimiş, C. (2015). A real-time life-care monitoring framework: WarnRed hardware and software design. Turkish Journal of Electrical Engineering \& Computer Sciences, 23, 1040-1050. https://doi.org/10.3906/elk-1304-178

Fanucci, L., Saponara, S., Bacchillone, T., Donati, M., Barba, P., Sanchez-Tato, I., \& Carmona, C. (2013). Sensing Devices and Sensor Signal Processing for Remote Monitoring of Vital Signs in CHF Patients. IEEE Transactions on Instrumentation and Measurement, 62(3), 553-569. https://doi.org/10.1109/ TIM.2012.2218681

Felipe Salech, M., Rafael Jara, L., \& Luis Michea, A. (2012). Cambios fisiológicos asociados al envejecimiento. Revista Médica Clínica Las Condes, 23(1), 19-29. https://doi.org/10.1016/S0716-8640(12)70269-9

Gómez García, C. A., \& Velasco Medina, J. (2014). Sistema de pulsioximetría y capnografía para dispositivos móviles Android. Ingeniería Biomédica, 8(15), 36-44. https://doi.org/10.24050/19099762.n15.2014.593

González, F., Villegas, O., Ramírez, D., Sánchez, V., \& Domínguez, H. (2014). Smart Multi-Level Tool for Remote Patient Monitoring Based on a Wireless Sensor Network and Mobile Augmented Reality. Sensors, 14(9), 17212-17234. https://doi.org/10.3390/s140917212

González Palacio, L., \& Urrego Giraldo, G. (2010). Modelo de contexto y de dominio para la ingeniería de requisitos de sistemas ubicuos. Revista Ingenierías Universidad de Medellin, 9(17), 151-164. Retrieved from https:// repository.udem.edu.co/handle/11407/779

Guevara Valdivia, M. E., Echegaray-Trelles, A., Hernández, J., Cordero-Pérez, L. de J., Valderrama de León, R., Santos, M. A., \& Huarte-Hernández, Y. (2011). Monitoreo remoto y seguimiento del paciente con desfibrilador automático implantable y terapia de resincronización cardiaca. Archivos de Cardiología de México, 81(2), 93-99.

Hassanalieragh, M., Page, A., Soyata, T., Sharma, G., Aktas, M., Mateos, G., ... Andreescu, S. (2015). Health Monitoring and Management Using Internet-of-Things (IoT) Sensing with Cloud-Based Processing: Opportunities and Challenges. In 2015 IEEE International Conference on Services Computing (pp. 285 292). IEEE. https://doi.org/10.1109/SCC.2015.47

Lopardo, G., Basombrío, A., Clara, L., Desse, J., De Vedia, L., Di Libero, E., ... Scapellato, P. (2015). Neumonia adquirida de la comunidad en adultos. Recomendaciones sobre su atención. Medicina, 75(4), 245-257.

Mileo, A., Merico, D., \& Bisiani, R. (2008). Wireless sensor networks supporting context-aware reasoning in assisted living. In Proceedings of the 1st ACM international conference on PErvasive Technologies Related to Assistive Environments - PETRA '08 (p. 1). New York, USA: ACM Press. https://doi. org/10.1145/1389586.1389651

Morelos Ramírez, R., Ramírez Pérez, M., Sánchez Dorantes, G., Chavarín Rivera, C., \& Meléndez-Herrada, E. (2014). El trabajador de la salud y el riesgo de enfermedades infecciosas adquiridas. Las precauciones estándar y de bioseguridad. Revista de La Facultad de Medicina UNAM, 57(4), 34-42. Retrieved from $\mathrm{http} / / /$ www.medigraphic.com/cgi-bin/new/resumen.cgi?IDARTICULO=51020

ORACLE. (2017). Oracle Advanced Analytics' Machine Learning Algorithms SQL Functions. Retrieved November 28, 2017, from https://www.oracle.com/technetwork/database/enterprise-edition/odm-techniquesalgorithms-097163.html

Organización de la Naciones Unidas. (2006). Convención sobre los derechos de las personas con discapacidad. Retrieved April 5, 2016, from http://www.un.org/spanish/disabilities/default.asp?id=497

Organización Mundial de la Salud. (2015). Informe Mundial sobre el envejecimiento y la salud. Retrieved from https://www.who.int/ageing/publications/world-report-2015/es/

Parra-Henao, G. J. (2010). Sistemas de información geográfica y sensores remotos. Aplicaciones en enfermedades transmitidas por vectores. CES Medicina, 24(2), 75-89.

Parra Alba, W. E. (2016). Modelo y sistema de análisis, generación y entrega de información, para apoyar la toma de decisiones a partir de datos obtenidos de pacientes remotos de la tercera edad con Neumonía Adquirida en la Comunidad (NAC). Pontificia Universidad Javeriana. Retrieved from https://repository.javeriana.edu. co/handle/10554/21196

Rajkomar, A., Mayer, A., \& Blandford, A. (2015). Understanding safety-critical interactions with a home medical device through Distributed Cognition. Journal of Biomedical Informatics, 56, 179-194. https://doi. org/10.1016/j.jbi.2015.06.002 
Sánchez, A., González, E., \& Barreto, L. (2018). Integrated Model AmI-IoT-DA for Care of Elderly People. In Advances in Computing (pp. 487-497). https://doi.org/10.1007/978-3-319-98998-3_37

Silva, J. M., Mouttham, A., \& El Saddik, A. (2009). UbiMeds: a mobile application to improve accessibility and support medication adherence. In Proceedings of the 1st ACM SIGMM international workshop on Media studies and implementations that help improving access to disabled users - MSIADU '09 (p. 71). New York, USA: ACM Press. https://doi.org/10.1145/1631097.1631109

Skubic, M., Guevara, R. D., \& Rantz, M. (2015). Automated Health Alerts Using In-Home Sensor Data for Embedded Health Assessment. IEEE Journal of Translational Engineering in Health and Medicine, 3, 1-11. https://doi.org/10.1109/JTEHM.2015.2421499

Sommerville, I. (1995). Software engineering. Retrieved from https://dl.acm.org/citation.cfm?id=211474

Villar-Montini, A. (2009). Remote wireless monitoring. Archivos de Cardiologia de Mexico, 79(2), 75-78.

Zamora-Izquierdo, M. A., Santa, J., \& Gomez-Skarmeta, A. F. (2010). An Integral and Networked Home Automation Solution for Indoor Ambient Intelligence. IEEE Pervasive Computing, 9(4), 66-77. https://doi. org/10.1109/MPRV.2010.20

\section{Sobre los autores}

\section{Andrés Armando Sánchez Martín}

Ingeniero de Sistemas de la Universidad Católica de Colombia (2012). Estudiante de la Maestría en Ingeniería de Sistemas y Computación de la Pontificia Universidad Javeriana. Se desempeña como profesor asistente en la Universidad de San Buenaventura. Realiza sus investigaciones en internet de las cosas (IoT), gestión y analítica de datos aplicados en domótica, tele-cuidado y producción agropecuaria.

\section{Enrique González Guerrero}

Ingeniero Eléctrico y Magíster en Ingeniería Eléctrica egresado de la Universidad de los Andes. En Francia obtuvo un D.E.A. en Robótica de la Universidad de París y un Doctorado en Informática de la Universidad de Evry. Investigador y docente de la Pontificia Universidad Javeriana desde 1999. Su trabajo se ha enfocado en la integración de los Sistemas Distribuidos y la Inteligencia Artificial, confluyendo hacia el desarrollo de Sistemas MultiAgentes. Los modelos conceptuales desarrollados y las herramientas generadas constituyen un marco para el análisis y síntesis de sistemas complejos, control inteligente de sistemas de robots cooperativos y aplicaciones con arquitecturas distribuidas basadas en agentes.

\section{Luis Eduardo Barreto Santamaría}

Profesional en Ingeniería de sistemas de la Universidad Católica de Colombia, Magíster en ingeniería de sistemas y computación de la Pontificia Universidad Javeriana, con más de 5 años de experiencia en el mercado laboral de las TIC en el sector educativo con el Ministerio de educación nacional, RENATA y Colciencias; en el sector del sistema general de pensiones con Colpensiones y en el sector de soluciones tecnológicas con casas de desarrollo de software. Asimismo, cuenta con casi 3 años de experiencia docente en la facultad de ingeniería de sistemas de la Universidad central y la universidad San Buenaventura en diversas materias de la línea de ciencias de la computación algorítmica, ingeniería de software, ingeniería web, programación, arquitectura orientada a servicios y gestión de proyectos informáticos. 